\title{
Enxerto aorto bi-ilíaco com veia femoral superficial: uma opção nas infecções de próteses e endopróteses aórticas - relato de caso
}

\author{
Aorto biiliac bypass with superficial femoral vein: an option in aortic grafts and endografts \\ infection - a case report
}

Patrick Bastos Metzger', Heraldo Antonio Barbato², Fernanda Maria Resegue Angelieri', Bruno Lourenção de Almeida', Frederico Augusto de Carvalho Linhares Filho', Eduardo da Silva Jordão', Antonio Massamitsu Kambara³, Fabio Henrique Rossi², Nilo Mitsuru Izukawa ${ }^{4}$

\begin{abstract}
Resumo
Uma das complicações mais temidas na cirurgia vascular é a infecção do enxerto sintético. Afeta 2\% dos procedimentos cirúrgicos convencionais e 0,3 a $6 \%$ dos procedimentos endovasculares. Relatamos o caso de paciente tratada de aneurisma de aorta abdominal por ambos os métodos e que, após seis anos, foi submetida a retirada das próteses devido à infecção. A veia femoral superficial bilateral foi usada como substituto, com sucesso.

Palavras-chave: Infecção; próteses e implantes; aneurisma.
\end{abstract}

\begin{abstract}
One of the most feared complications in vascular surgery is the infection of synthetic graft. It affects $2 \%$ of conventional surgery procedures and about 0.3 to $6 \%$ of endovascular procedures. We report the case of a patient treated for abdominal aortic aneurysm with both methods; after six years, this patient had the prosthesis removed because of infection. Bilateral superficial femoral vein were used as substitute with success.
\end{abstract}

Keywords: Infection; prostheses and implants; aneurysm.

\section{Introdução}

Uma das complicações mais temidas na cirurgia vascular é a infecção de enxerto com prótese sintética. Ocorre em torno de $2 \%$ dos procedimentos e pode provocar deiscência das suturas arteriais, promovendo hemorragias de difícil controle, fístulas entre a prótese e as alças intestinais, fistulas aortoentéricas (FAE) e, se não resolvidas em tempo hábil, são invariavelmente fatais ${ }^{1-5}$.

Os procedimentos endovasculares para implante de endoprótese aórtica apresentam incidência de infecção que pode variar de 0,3 a $6 \%$. Em trabalho de revisão de literatura, foram identificados 62 casos de infecção de próteses endovasculares aortoilíacas, perfazendo incidência de $0,4 \%{ }^{5}$.

Em caso de infecção de prótese, o objetivo do tratamento consiste habitualmente em remover o enxerto infectado e restabelecer a continuidade vascular com derivações extraanatômicas ou novo enxerto in situ ${ }^{6}$. A partir da década de 1980, a cirurgia com bypass extra-anatômico e retirada total do enxerto infectado foi o tratamento mais utilizado $0^{7-9}$. Essa técnica apresenta taxa de infecção entre 5 a $8 \%^{1-5,10}$. Recentemente, a substituição do material infectado por enxertos venosos in situ tem sido empregada com sucesso, com a vantagem de ser material autógeno e diminuir a chance de sangramento da sutura no coto aórtico remanescente, com

Trabalho realizado no Instituto Dante Pazzanese de Cardiologia, São Paulo (SP), Brasil.

'Médicos Residentes da Seção Médica de Cirurgia Vascular do Instituto Dante Pazzanese de Cardiologia, São Paulo (SP), Brasil.

${ }^{2}$ Cirurgião Vascular da Seção Médica de Cirurgia Vascular do Instituto Dante Pazzanese de Cardiologia, São Paulo (SP), Brasil.

${ }^{3}$ Chefe da Seção Médica de Radiologia do Instituto Dante Pazanesse de Cardiologia, São Paulo (SP), Brasil.

${ }^{4}$ Chefe da Seção Médica de Cirurgia Vascular do Instituto Dante Pazzanese de Cardiologia, São Paulo (SP), Brasil.

Não foram declarados conflitos de interesse associados à publicação deste artigo.

Submetido em: 23.08.2010. Aceito em: 12.04.2011

J Vasc Bras. 2011;10(2):160-164. 
perspectiva de maior taxa de salvamento de membro ${ }^{11,12}$. A utilização da veia femoral superficial para enxerto arterial foi inicialmente descrita por Schulman ${ }^{13-15}$ no território femoropoplíteo. A partir de 1997, foram publicadas pequenas séries de casos, em outros territórios arteriais, com resultados satisfatórios ${ }^{12,16,17 .}$

Apesar dos grandes avanços do peri e pós-operatórios e da terapia antimicrobiana, a taxa de mortalidade e morbidade operatória do tratamento das infecções de próteses e endopróteses aórticas (IPEA) permanece elevada ${ }^{6}$.

\section{Relato de caso}

Trata-se de paciente de 64 anos, sexo feminino, com história de correção de Aneurisma de Aorta Abdominal (AAA) infrarrenal, submetida a enxerto aorto-aórtico com prótese de Dacron de $23 \mathrm{~mm}$, sem intercorrências. Após 9 anos, apresentou dilatação do colo proximal da aorta infrarrenal com diâmetro máximo de $43 \mathrm{~mm}$. Foi submetida a correção endovascular com colocação de endoprótese revestida tubular aorto-biíliaca Apolo-Nano endoluminal de $28 \times 14$ x $150 \mathrm{~mm}$. A via de acesso foi a artéria femoral comum, por dissecção bilateral. O procedimento foi realizado com sucesso, sem intercorrências e sem vazamento na arteriografia de controle.

Retornou após seis anos, com história de sete dias de dor lombar com irradiação para fossa ilíaca esquerda, associada a febre, anorexia e queda do estado geral. No exame

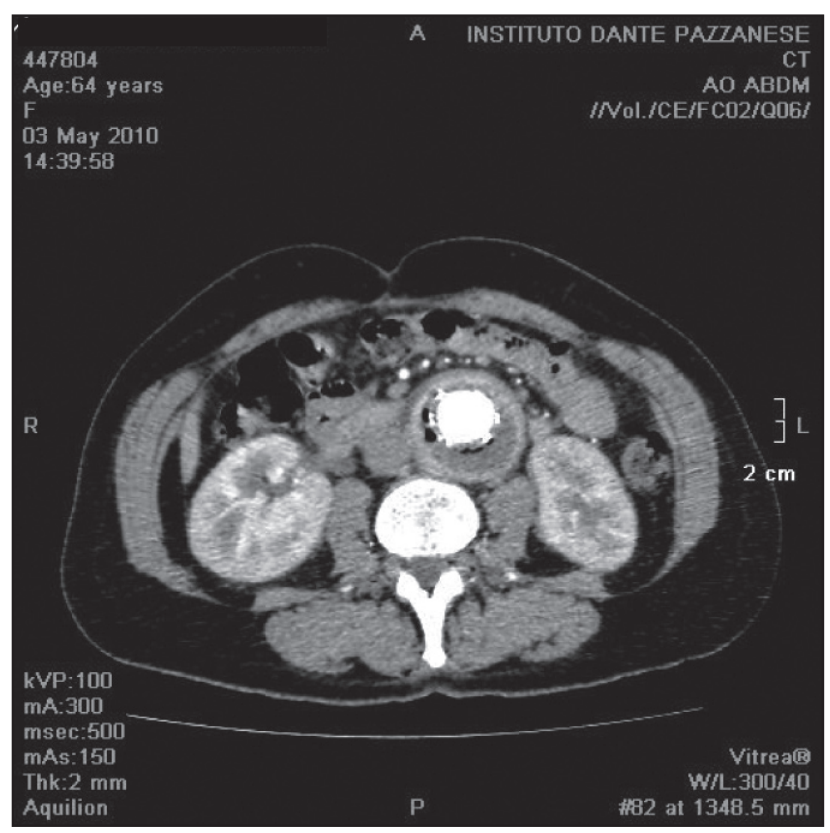

Figura 1 - Tomografia de abdomen com coleção gasosa periprótese aórtica. físico, apresentava abdomen doloroso à palpação profunda, sem sinais de irritação peritoneal. Apresentava exames laboratoriais dentro da normalidade. Realizada tomografia computadorizada, que evidenciou presença de coleção gasosa e líquida periprótese aórtica (Figuras 1 e 2), confirmando a infecção de prótese e endoprótese aórtica. Iniciada antibioticoterapia com Tazobactam + Vancomicina. A hemocultura e a urocultura colhidas na admissão foram negativas para fungos e bactérias.

Os antibióticos foram mantidos por 28 dias sem melhora clínica, persistindo quadro de febre diária e calafrios esporádicos.

A paciente foi submetida, então, à cirurgia para retirada de prótese e endoprótese aórtica com reconstrução in situ, utilizando-se veia femoral superficial bilateral. Foi realizado enxerto aortoilíaco comum à direita e ilíaco externo à esquerda (Figuras 3 e 4). No mesmo ato, foi realizado desbridamento extenso e lavagem copiosa do retroperitônio, com solução salina. Ainda durante o intraoperatório, foram observadas aderências enteroaórticas que foram desfeitas e suturadas primariamente (Figura 5).

A evolução pós-operatória foi satisfatória, apresentando-se afebril durante todo o período, com pulsos normais em membros inferiores, sem edema e sem leucocitose. $\mathrm{O}$ uso de vancomicina e tazobactam foi mantido por cinco

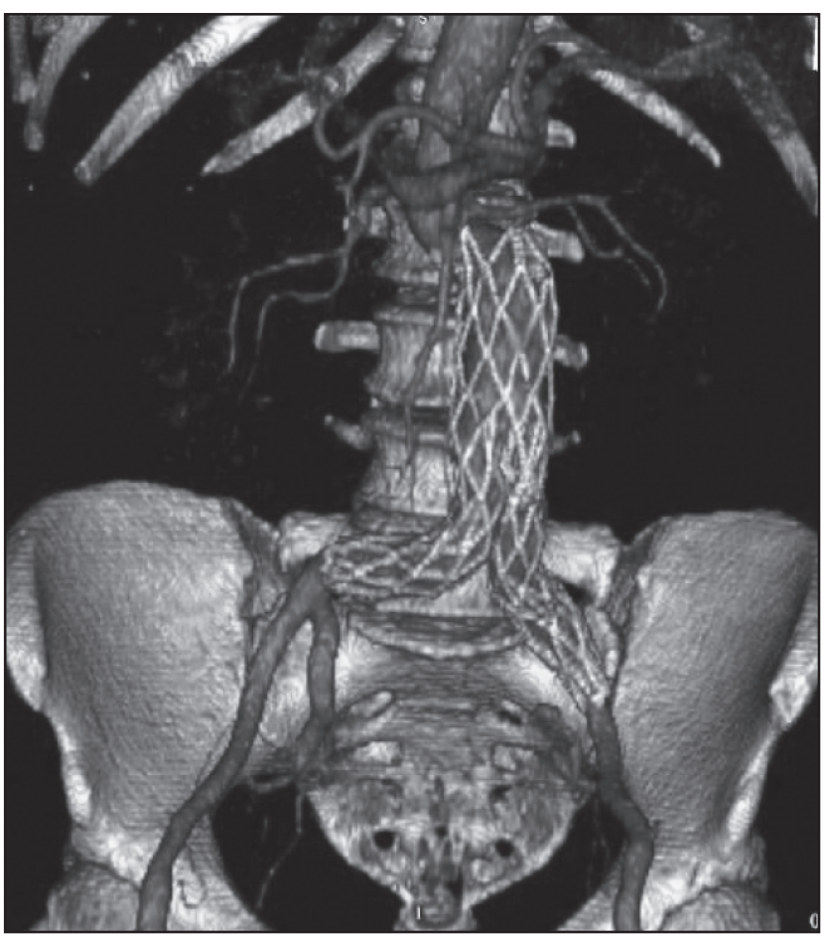

Figura 2 - Reconstrução em três dimensões da tomografia de abdomen mostrando seu colo proximal e oclusão da artéria hipogástrica esquerda. 
dias no pós-operatório. Recebeu alta hospitalar no sétimo dia pós-operatório com prescrição apenas de estatina e ácido acetil salicílico. Atualmente, encontra-se em acompanhamento ambulatorial, com evolução favorável. Nova angiotomografia de controle demonstrou enxerto pérvio e sem sinais de infecção.

A cultura de secreção colhida durante o intraoperatório e semeada em Agar sangue chocolate e MacConckey teve resultado negativo para crescimento de bactérias e fungos após 48 horas de cultivo.

A cultura da prótese e endoprótese aórtica também foi negativa para bactérias e fungos após sete dias.

$\mathrm{O}$ exame anatomopatológico da peça retirada na cirurgia mostrou extensa reação inflamatória e supurativa, com presença de múltiplos cocos gram-positivos (Figuras 6 e 7).

\section{Discussão}

A retirada de prótese aórtica infectada e reconstrução do fluxo arterial com enxerto axilobifemoral é a cirurgia tradicional para as IPEA, apesar de as taxas de morbidade e mortalidade não serem baixas ${ }^{1,2,10}$. Observa-se atualmente o uso crescente de reconstruções in situ com materiais autógenos, sendo a veia femoral superficial um dos condutos preferenciais devido ao seu maior diâmetro, à sua maior

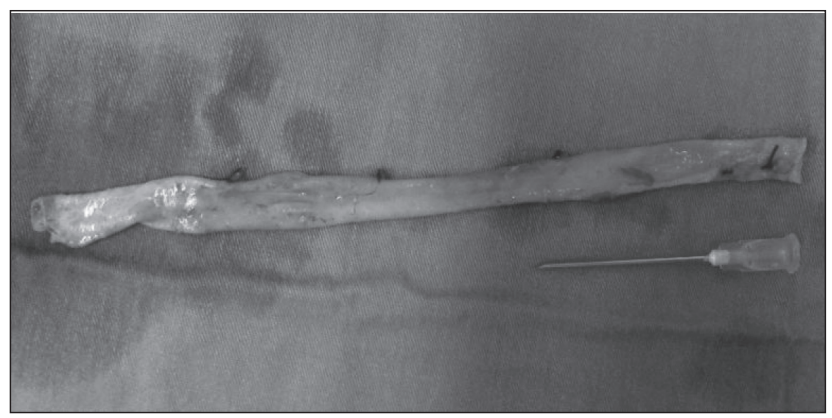

Figura 3 - Conduto venoso femoral superficial.

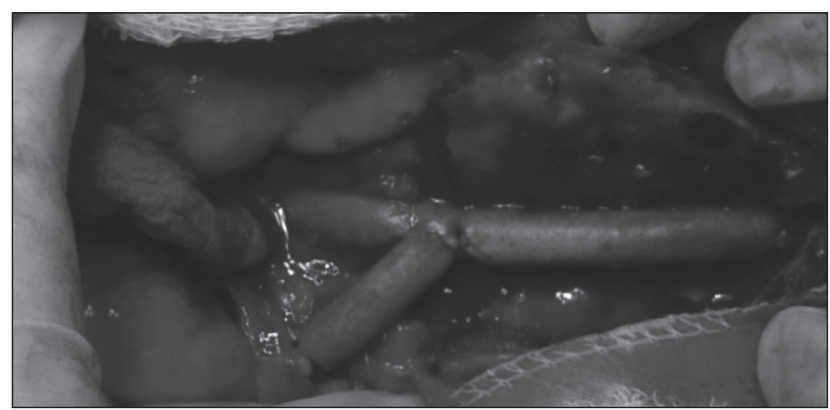

Figura 4 - Enxerto aorto bi-ilíaco com veia femoral superficial.

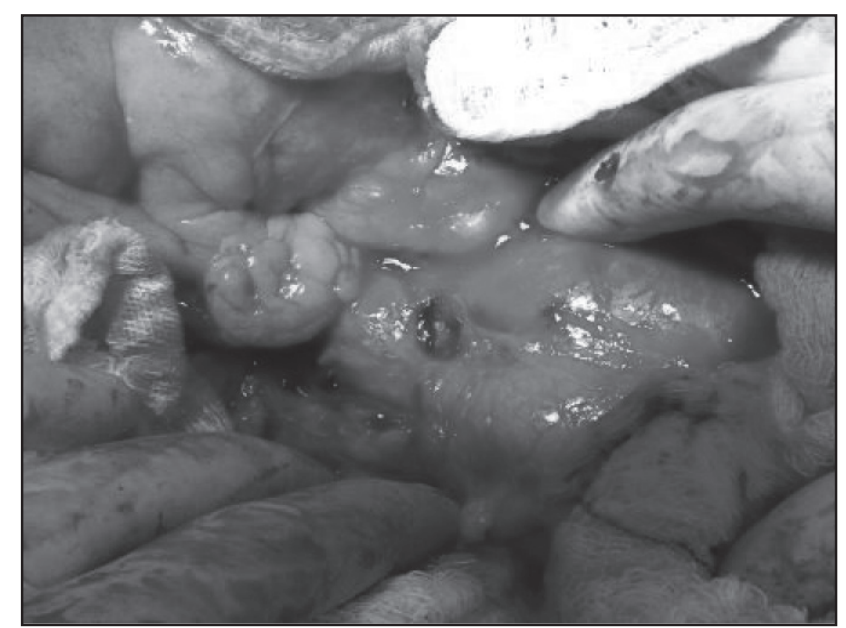

Figura 5 - Aderência enteroaórtica.

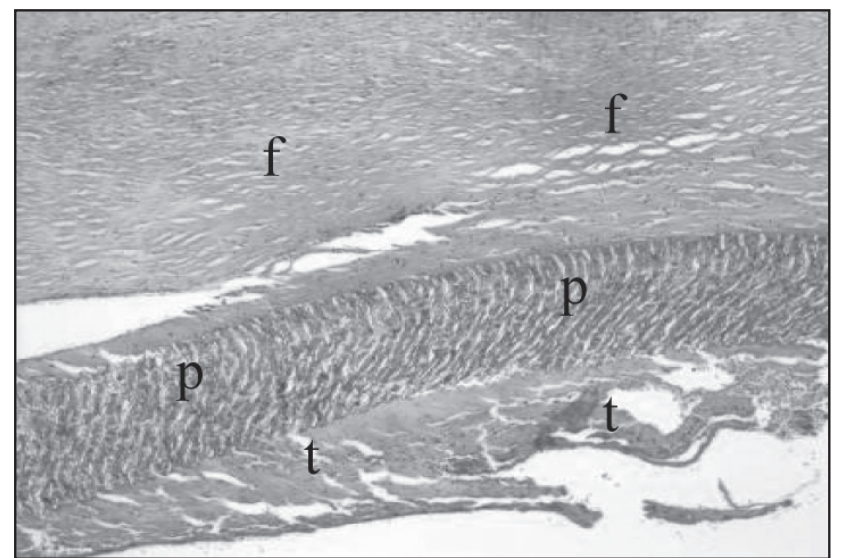

Figura 6 - Faixa de material protético (p) com uma face revestida por espessa capa fibrosa densa (f). Na face oposta, depositam-se trombos (t) que englobam colônias bacterianas e células inflamatórias.

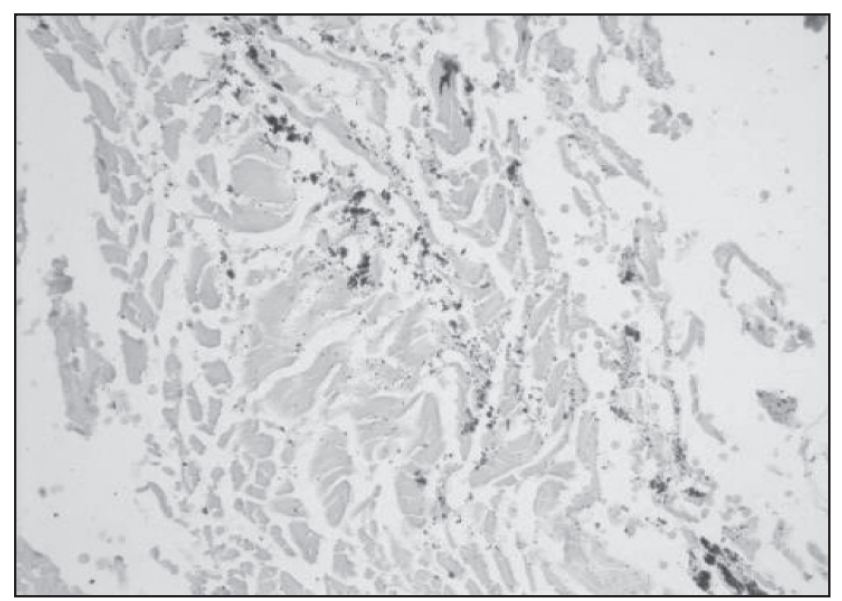

Figura 7 - Colônias de cocos gram-positivos em meio a fibrina e tecido necrótico. Coloração de Gram para tecido (1.000x). 
resistência a infecção, além de possuir superficie não trombogênica, apropriada para as revascularizações arteriais ${ }^{6}$.

$\mathrm{O}$ uso da veia safena magna no território aortofemoral tem apresentado resultado pobre, provavelmente devido à hiperplasia intimal e seu baixo calibre, com taxas de perviedade de apenas $40 \%$ em algumas casuísticas ${ }^{18,19}$. Por outro lado, os condutos utilizando as veias femoral superficial e poplítea apresentam baixa incidência de desenvolvimento de estenoses e baixo potencial de degeneração aneurismática ${ }^{20}$.

Ali et al., em acompanhamento de 187 pacientes submetidos a reconstruções aórticas com enxertos autógenos de seguimentos venosos femoropoplíteos, demonstraram peviedade primária assistida e secundária de 81 e 91\%, respectivamente, em 7 anos. A taxa de mortalidade até 30 dias foi de $10 \%$ e a sobrevida em 1 ano, de $87 \%$, com taxa de salvamento de membro de $92,6 \%{ }^{6}$. Destacaram ainda como fatores preditores independentes de mortalidade a idade e as complicações gastrointestinais, especialmente as $\mathrm{FAE}^{6,21,22}$.

Enxertos sintéticos embebidos com rimfampicina têm sido relatados na literatura com bons resultados em infecções pouco virulentas e limitadas a infecções de S. epidermidis, apesar do baixo seguimento desses pacientes ${ }^{11}$.

Estudo recente sugere que o uso de próteses revestidas com prata pode ser tão efetivo e seguro quanto o uso de aloenxertos arteriais, associado a um menor custo. Esta deve ser uma opção nos pacientes que não possuem enxertos homólogos disponíveis ${ }^{23}$.

No caso de nossa operação, observamos aderências aortoentéricas que foram desfeitas e suturadas primariamente. Não foi observada qualquer complicação gastrointestinal durante o pós-operatório. As experiências de alguns autores na literatura mostram que a reconstrução gastrointestinal é um determinante primário do desfecho dessa cirurgia e preconizam o isolamento do reparo das FAE com interposição de omento, para melhor isolamento das alças intestinais com o novo enxerto ${ }^{6}$.

O insucesso terapêutico dessas reconstruções está diretamente relacionado a algumas variáveis, como perda sanguínea maior que $3 \mathrm{~L}$ no intraoperatório, presença de insuficiência renal, risco clínico elevado (escore preconizado pela Sociedade Americana de Anestesia - ASA > 4 ) e presença de sepse $e^{5,6}$.

Na operação de nossa paciente, houve perda de 1.300 $\mathrm{mL}$ de sangue sem descompensação hemodinâmica durante o intra e pós-operatório, apresentando diurese satisfatória e mantendo níveis de creatinina sérica dentro da normalidade.
Uma manobra cirúrgica importante nesses casos é o desbridamento extenso dos tecidos aórticos e periaórticos infectados, assim como a lavagem copiosa com solução salina do leito de implante do novo enxerto. O tempo estimado dessas reconstruções varia de 6 a 11 horas segundo a literatura, sendo que a nossa cirurgia foi realizada em 6 horas $^{21}$.

Quanto ao perfil bacteriológico das IPEAs, sabe-se que o Staphylococcus aureus é o agente etiológico preponderante nos casos das infecções de prótese, principalmente as precoces, enquanto que o Staphylococcus epidermidis tem apresentado aumento de incidência nas infecções mais tardias, no decorrer de vários meses e até anos. Além disso, devido a sua menor agressividade, são comumente associadas a culturas negativas da prótese, dos tecidos e das secreções periaórticas ${ }^{5}$. As infecções por bactérias anaeróbicas são mais graves, sendo associadas a sepsis e toxicidade sistêmica severa ${ }^{6}$.

Infeções fúngicas por Candida glabrata estão associadas a curso prolongado de múltiplos antibióticos, apresentando-se clinicamente como infecções crônicas, ocasionando sinais de desnutrição e debilidade geral. Está associada, frequentemente, à FAE e a desfechos desfavoráveis. Infecções por Pseudomonas aeruginosa e Staphylococcus aureus meticilina-resistentes são graves e podem estar vinculadas à ruptura aguda ou deiscência da linha de sutura dos enxertos com material autólogo ${ }^{24}$.

Em nosso caso, as culturas das secreções peri-prótese, além das culturas da prótese de Dacron e da endoprótese aórtica, mostraram-se negativas. Esse fato pode ser explicado devido à baixa virulência do micro-organismo e ao curso prolongado de antibióticos recebido pela paciente durante o período pré-operatório, uma vez que foram encontrados múltiplos cocos gram-positivos no exame anatomopatológico que não cresceram em meio de cultivo apropriado. Dados da literatura demostram que 15\% das culturas das IPEAs são negativas para o crescimento de micro-organismos ${ }^{6}$.

A piora da insuficiência venosa crônica após a retirada da veia femoral superficial tem se mostrado pouco prevalente nas descrições dos estudos clínicos, uma vez que a perviedade da veia femoral comum, profunda e poplítea infra-articular mantém o retorno venoso do membro acometido, não havendo edema importante ou aumento significativo da estase venosa ${ }^{25}$.

As veias femorais superficiais são adequadas para reconstruções aorto-femorais, porém não devem ser utilizadas nos pacientes com histórico de trombose venosa profunda. Apesar disso, até $80 \%$ dos pacientes com IPEA são candidatos a esse tipo de reconstrução ${ }^{19}$. 


\section{Conclusão}

Consideramos a reconstrução aortoilíaca com veia femoral superficial autógena uma boa opção nas IPEAs, pois a veia femoral superficial, além de ser um bom conduto, tem maior resistência à infecção; há também o fato de apresentar boa perviedade a longo prazo e taxa de morbimortalidade comparável aos enxertos extra-anatômicos.

\section{Referências}

1. Lawrence PF. Management of infected aortic grafts. Surg Clin North Am. 1995;75:783-97.

2. Cairols MA, Simeon JM, Guerrero F, et al. Infected grafts require excision and extra-anatomic reconstruction: for the motion. In: Greenhalgh RM, editor. The evidence for vascular or endovascular reconstruction. Philadelphia: W.B. Saunders; 2002. p. 235-51.

3. Sladen JG, Chen JC, Reid JD. An aggressive local approach to vascular graft infection. Am J Surg. 1998;176:222-5.

4. Hannon RJ, Wolfe JH, Mansfield AO. Aortic prosthetic infection: 50 patients treated by radical or local surgery. $\mathrm{Br}$ J Surg. 1996;83:654-58.

5. Frankini AD, Cardozo MA, Lichtenfels E. Infecção em prótese vascular. In: Brito J, editor. Cirurgia Vascular, Endovascular e Angiologia. Rio de Janeiro: Revinter; 2006. p. 866-83.

6. Ali AT, Modrall JG, Hocking J, et al. Long-term results of the treatment of aortic graft infection by in situ replacement with femoral popliteal vein grafts. J Vasc Surg. 2009;50:30-9.

7. Reilly LM, Stoney RJ, Goldstone J, Ehrenfeld WK. Improved management of aortic graft infection: the influence of operation sequence and staging. I Vasc Surg. 1987;5:421-31.

8. Seeger JM, Pretus HA, Welborn MB, Ozaki CK, Flynn TC, Huber TS. Long-term outcome after treatment of aortic graft infection with staged extra-anatomic bypass grafting and aortic graft removal. J Vasc Surg. 2000;32:451-61.

9. Yeager RA, Taylor LM, Moneta GL, et al. Improved results with conventional management of infrarenal aortic infection. J Vasc Surg. 1999;30:76-83.

10. O'Connor S, Andrew P, Batt M, Becquemin JP. A systematic review and meta-analysis of treaments for aortic graft infection. J Vasc Surg. 2006;44:38-45.

11. Bandyk DF, Kinney EV, Riefsnyder TI, Kelly H, Towne JB. Treatment of bacteria-biofilm graft infection by in situ replacement in normal and immune-deficient states. J Vasc Surg. 1993;18:398-405.

12. Bandyk DF, Novotney ML, Back MR, Johnson BL, Schmacht DC. Expanded application of in situ replacement for prosthetic graft infection. J Vasc Surg. 2001;34:411-20.

13. Schulman ML, Badhey MR, Yatco R. Superficial femoral-popliteal veins and reversed saphenous veins as primary femoropopliteal bypass grafts: a randomized comparative study. I Vasc Surg. 1987;6:1-10.
14. Schulman ML, Badhey MR, Yatco R, Pillari G. An 11-year experience with deep leg veins as femoropopliteal bypass grafts. Arch Surg. 1986;121:1010-5.

15. Schulman ML, Schulman LG. Deep leg veins as femoropopliteal bypass grafts. World J Surg. 1990;14:843-5.

16. Clagett GP, Bowers BL, Lopez-Viego MA, Rossi MB, Valentine RJ, Myers SI, et al. Creation of a neo-aortoiliac system from lower extremity deep and superficial veins. Ann Surg. 1993;218:239-48.

17. Clagett GP, Valentine RJ, Hagino RT. Autogenous aortoiliac/femoral reconstructin from superficial femoral-popliteal veins: feasibility and durability. J Vasc Surg. 1997;25:255-66.

18. Seeger JM, Wheeler JR, Gregory RT, Snyder SO, Gayle RG. Autogenous graft replacement of infected prosthetic grafts in the femoral position. Surgery. 1983;93:39-45.

19. Clagett GP, Bowers BL, Lopez-Viego MA, et al. Creation of a neoaortoiliac system from lower extremity deep and superficial veins. Ann Surg. 1993;218:239-49.

20. Beck AW, Murphy EH, Hocking JA, Timaran CH, Arko FR, Clagett GP. Aortic reconstruction with femoral popliteal vein: graft stenosis incidence, risk and reintervention. J Vasc Surg. 2008;47:36-43.

21. Valentine R, Timaran CH, Modrall G), Smith ST, Arko FR, Clagett GP. Secondary aortoenteric fistulas versus paraprosthetic erosions: is bleeding associated with worse outcome? ) Am Coll Surg. 2008;207:922-7.

22. Ali AT, McLeod N, Kalapatapu VR, Moursi MM, Eidt JF. Staging the neoaortioiliac system: feasibility and short-term outcomes. J Vasc Surg. 2008;48:1125-30

23. Pupka A, Skora J, Janczak D, Plonek T, Marczak J, Szydełko T. In situ revascularisation with silver-coated polyester protheses and arterial homografts in patients with aortic graft infection--a prospective, comparative, single- centre study. Eur J Vasc Endovasc Surg. 2011;41:61-7.

24. Speziale F, Rizzo L, Sbarigia E, et al. P. Bacterial and clinical criteria relating to the outcome of patients undergoing in situ replacement of infected abdominal aortic grafts. Eur J Vasc Endovasc Surg. 1997;13:127-33.

25. Modrall JG, Hocking JA, Timaran $\mathrm{CH}$, et al. Late incidence of chronic venous insufficiency after deep vein harvest. J Vasc Surg. 2007:46:520-5.

Correspondência: Patrick Bastos Metzger Rua Maestro Callia, 271 - Vila Mariana CEP 04012-100 - São Paulo (SP), Brasil E-mail: patrickvascular@gmail.com

Contribuições dos autores: Concepção e desenho do estudo: PBM, NMI, HAB. Análise e interpretação dos dados: PBM, NMI, FHR, AMK Coleta de dados: PBM, FOL, FMRA Redação do artigo: PBM, FMRA Revisão crítica do texto: PBM, NMI, AMK Aprovação final do artigo*: PBM, HAB, FMRA, FOL, BLA, ESJ, FHR, AMK, NMI Análise estatística: PBM, ESJ, BLA Responsabilidade geral pelo estudo: PBM, NMI Informações sobre financiamento: PBM, NMI * Todos os autores leram e aprovaram a versão final submetida ao J Vasc Bras. 